\title{
INFLUENCING OF LAW OF TRAFFIC CONTROL OF SHIP ON COURSE ON EXACTNESS OF NAVIGATION
}

\section{ВЛИЯНИЕ ЗАКОНА УПРАВЛЕНИЯ ДВИЖЕНИЕМ СУДНА ПО КУРСУ НА ТОЧНОСТЬ СУДОВОЖДЕНИЯ}

\author{
V.E. Sikirin, senior lecturer \\ B.Е. Сикирин, старший преподаватель \\ National University «Odessa Maritime Academy», Ukraine \\ Национальный университет «Одесская морская академия», Украина
}

\begin{abstract}
Differential equalization of the guided motion of ship is resulted on the angle of prowling. Depending on different types of management of ship equalization of change of angle of his prowling is got. It is shown that the lateral taking of ship depends on descriptions of moments of indignation, and also from frequency of own vibrations of ship after the angle of prowling and his coefficient of fading. Analytical expressions of maximal value of the lateral tearing down of ship are got at proportionally-differential and proportsyonal - integral - differential algorithm of management of ship.

In work differential equalization of motion of ship is resulted on the angle of prowling which is determined by the moment of inertia of ship and added the masses of water in relation to vertical to the landmark, by the hydrodynamic moment of resistance, moment from a helm and revolting moment.

The got differential equalization is heterogeneous linear with permanent coefficients, the common decision of which consists of decision of the proper homogeneous equalization and partial decision which is determined by right part of differential equalization.

Own attenuation vibrations of ship on the angle of prowling are the decision of the proper homogeneous equalization, the period of which depends from a moment inertia of ship in relation to vertical to the landmark, and also from a hydrodynamic coefficient and coefficient of feather of helm of ship.

The partial decision characterizes taking which is the sum of two constituents: taking of ship under action of permanent constituent of revolting moment and the forced oscillation of ship on the angle of prowling which arises up through the harmonic constituent of revolting moment. For indemnification of permanent displacement from the asymmetrical prowling an additional signal what proportional to the integral is entered in the law of management, and also a signal dependency upon angular speed of prowling is used. He diminishes amplitude of prowling of ship in relation to the programmatic course of ship.

In completion of the article dependence of exactness of realization by the ship of programmatic trajectory is considered on the law of traffic control of ship on a
\end{abstract}


course. It is shown that most exactness of maintenance of ship on the set trajectory is provided PID the law of management, so as warns appearance of permanent displacement and provides the minimum value of amplitude of prowling of the forced vibrations.

Keywords: safety of navigation, exactness of realization of program trajectory, law of management of motion of ship.

\section{PЕФЕРАТ}

Наведено диференційне рівняння керованого руху судна по куту рискання. Залежно від різних типів управління судна отримано рівняння зміни кута його рискання. Показано, що бічне знесення судна залежить від характеристик моментів збурення, а також від частоти власних коливань судна за кутом рискання i його коефіцієнту загасання. Отримано аналітичні вирази максимального значення бокового зносу судна при пропорційнодиференційному i пропорційно-інегрально-диференційному алгоритму керування судна.

В роботі приведено диференційне рівняння руху судна по куту рискання, яке визначається моментом інерції судна i приєднаних мас води відносно вертикальної вісі, гідродинамічним моментом супротиву, моментом від керма та збурюючим моментом.

Одержане диференційне рівняння являється неоднорідним лінійним 3 постійними коефіцієнтами, загальне рішення якого складається 3 рішення відповідного однорідного рівняння та часткового розв'язку, який визначається правою частиною диференційного рівняння.

Рішенням відповідного однорідного рівняння являються власні затухаючі коливання судна по куту рискання, період яких залежить від моменту інерції судна відносно вертикальної вісі, а також від гідродинамічного коефіцієнта та коефіцієнта пера керма судна.

Частковий розв'язок характеризує знесення, яке являється сумою двох складових: знесення судна під дією постійної складової збурюючого моменту та вимушене коливання судна по куту рискання, яке виникає через гармонічну складову збурюючого моменту. Для компенсації постійного зміщення від несиметричних рискань в закон управління вводиться додатковий сигнал, який пропорційний інтегралу, а також використовується сигнал, залежний від кутової швидкості рискань. Він зменшує амплітуду рискань судна відносно програмного курсу судна.

В завершення статті розглянуто залежність точності реалізації судном програмної траєкторії від закону управління рухом судна по курсу. Показано, що найбільшу точність утримання судна на заданій траєкторії забезпечується ПІД законом управління, так як попереджує появу постійного зміщення i забезпечує мінімальне значення амплітуди рискання вимушених коливань.

Ключові слова: безпека судноводіння, точність реалізації програмної траєкторії, закони управління руху судна. 
Постановка проблемы в общем виде и ее связь с важными научными или практическими задачами

Снижение аварийности судов при плавании в стесненных водах повышает безопасность судовождения. Решению этой проблемы способствует повышение точности реализации судном программной траектории движения, причем точность в определяющей мере зависит от закона управления движения судна по курсу. Поэтому следует исследовать влияние закона управления движения судна по курсу на точность реализации судном программной траектории, чему посвящена данная статья.

Анализ последних достижений и публикаций, в которых начато решение данной проблемы и выделение нерешенных ранее частей общей проблемы

Как указывается в работе [1], в настоящее время производится разработка информационной системы имитационного моделирования движения судов со сложными динамическими моделями, в зависимости от угла кладки руля и оборотов двигателя. Данная система позволит обеспечить новый тип планирования маневров судна и контроль реализации заданного маневра. Предусмотрено в процессе маневрирования отображать заданные маневры одновременно с фактическим движением судна и с индикацией прогнозируемой траекторией, которая определяется реальными входными данными от датчиков судна.

Работа [2] посвящена вопросам идентификации судовых моделей маневрировании, которые являются ключом к исследованию маневренности судна, проектированию систем управления движением судов и развитию систем управления судовыми тренажерами. В данной работе на основе анализа гидродинамики судна сформирована нелинейная модель маневрирования судна.

В работе [3] автор рассматривает интеллектуальную систему прогнозирования движения судна, которая имитирует процесс обучения автономного блока управления, созданного с помощью искусственной нейронной сети. Блок управления наблюдает входные сигналы и вычисляет значения требуемых параметров маневрировании судна в стесненных водах. Основной задачей системы является непрерывный контроль навигационных параметров судна и прогноз их значений после определенного интервала времени.

\section{Формулировка целей статьи}

Целью статьи является исследование зависимости точности движения судна программной траекторией от закона управления движения судна по курсу.

\footnotetext{
Изложение основного материала исследования с обоснованием полученных научных результатов

В первом приближении дифференциальное уравнение движения судна по углу рыскания $\psi$ имеет вид:
} 


$$
\mathrm{I}_{\mathrm{z}} \ddot{\psi}+\mathrm{C}_{1} \dot{\psi}+\mathrm{C}_{2} \beta=\mathrm{L},
$$

где $\mathrm{I}_{\mathrm{z}}$ - момент инерции судна и присоединенных масс воды относительно вертикальной оси;

$\mathrm{C}_{1}$ - гидродинамическая характеристика судна;

$\mathrm{C}_{2}$ - гидродинамическая характеристика руля;

$\mathrm{L}$ - возмущающий момент.

Возмущающий момент $\mathrm{L}$ является суммой двух составляющих: постоянной $\mathrm{L}_{\mathrm{c}}$ и гармонической $\mathrm{L}_{\mathrm{p}}$, т. е.:

$$
\mathrm{L}=\mathrm{L}_{\mathrm{c}}+\mathrm{L}_{\mathrm{p}}=\mathrm{L}_{\mathrm{c}}+\mathrm{L}_{\mathrm{po}} \sin \omega_{\mathrm{p}} \mathrm{t},
$$

где $\mathrm{L}_{\mathrm{po}}$ и $\omega_{\mathrm{p}}$ - соответственно амплитудное значение гармонической составляющей и круговая частота ее изменения.

С учетом этого дифференциальное уравнение (1) принимает вид:

$$
\mathrm{I}_{\mathrm{z}} \ddot{\psi}+\mathrm{C}_{1} \dot{\psi}+\mathrm{C}_{2} \beta=\mathrm{L}_{\mathrm{c}}+\mathrm{L}_{\mathrm{po}} \sin \omega_{\mathrm{p}} \mathrm{t} .
$$

Разделяя на коэффициент при старшей производной в данном уравнении, получим:

$$
\ddot{\psi}+\frac{\mathrm{C}_{1}}{\mathrm{I}_{\mathrm{z}}} \dot{\psi}+\frac{\mathrm{C}_{2}}{\mathrm{I}_{\mathrm{z}}} \beta=\frac{\mathrm{L}_{\mathrm{c}}}{\mathrm{I}_{\mathrm{z}}}+\frac{\mathrm{L}_{\mathrm{po}}}{\mathrm{I}_{\mathrm{z}}} \sin \omega_{\mathrm{p}} \mathrm{t} .
$$

Угол кладки руля $\beta$ зависит от закона управления судном. В случае пропорционального закона управления угол $\beta$ линейно зависит от угла рыскания судна $\psi$, т. е. $\beta=\frac{\mathrm{k}_{1}}{\mathrm{k}_{4}} \psi$, где $\mathrm{k}_{1}$ и $\mathrm{k}_{4}$ - коэффициенты.

В этом случае уравнение (2) принимает вид:

$$
\ddot{\psi}+2 v_{\theta} \dot{\psi}+\omega_{\mathrm{o}}^{2} \psi=\frac{\mathrm{L}_{\mathrm{c}}}{\mathrm{I}_{\mathrm{z}}}+\frac{\mathrm{L}_{\mathrm{po}}}{\mathrm{I}_{\mathrm{z}}} \sin \omega_{\mathrm{p}} \mathrm{t},
$$

где $v_{\theta}=\frac{\mathrm{C}_{1}}{2 \mathrm{I}_{\mathrm{z}}}-$ коэффициент затухания;

$\omega_{\mathrm{o}}^{2}=\frac{\mathrm{C}_{2}}{\mathrm{I}_{\mathrm{z}}} \frac{\mathrm{k}_{1}}{\mathrm{k}_{4}}-$ собственная частота судна при рыскании.

Дифференциальное уравнение (3) является неоднородным линейным с постоянными коэффициентами. Найдем решение дифференциального уравнения (3), которое описывает рыскание судна. Согласно [4] решение будем искать в следующей форме:

$$
\psi=\psi_{\mathrm{ob}}+\psi_{\mathrm{r}},
$$

где $\psi_{\mathrm{ob}}$ - решение соответствующего (3) однородного уравнения;

$\psi_{\mathrm{r}}$ - частное решение, определяющееся правой частью уравнения (3).

Найдем частное решение $\psi_{\mathrm{r}}$ дифференциального уравнения (3), которое описывает движение судна при действии возмущающего момента L. Учитывая 
структуру возмущающего момента L, согласно [4] частное решение будем искать в следующей форме:

$$
\psi_{\mathrm{r}}=\mathrm{P}_{\mathrm{o}}+\mathrm{P}_{1} \sin \omega_{\mathrm{p}} \mathrm{t}+\mathrm{P}_{2} \cos \omega_{\mathrm{p}} \mathrm{t}
$$

где требуется определить неизвестные коэффициенты $\mathrm{P}_{\mathrm{o}}, \mathrm{P}_{1}$ и $\mathrm{P}_{2}$. Для этого необходимо найти первую и вторую производные угла рыскания $\psi$ и подставить их в уравнение (3), после чего получим:

$$
\begin{gathered}
-P_{1} \omega_{p}^{2} \sin \omega_{p} t-P_{2} \omega_{p}^{2} \cos \omega_{p} t+2 \nu_{\theta}\left(P_{1} \omega_{p} \cos \omega_{p} t-P_{2} \omega_{p} \sin \omega_{p} t\right)+ \\
+\omega_{o}^{2}\left(P_{o}+P_{1} \sin \omega_{p} t+P_{2} \cos \omega_{p} t\right)=\frac{L_{c}}{I_{z}}+\frac{L_{p o}}{I_{z}} \sin \omega_{p} t
\end{gathered}
$$

Группируем члены в левой части уравнения:

$$
\begin{gathered}
\left(-\mathrm{P}_{1} \omega_{\mathrm{p}}^{2}-2 v_{\theta} \mathrm{P}_{2} \omega_{\mathrm{p}}+\omega_{\mathrm{o}}^{2} \mathrm{P}_{1}\right) \sin \omega_{\mathrm{p}} \mathrm{t}+\left(-\mathrm{P}_{2} \omega_{\mathrm{p}}^{2}+2 v_{\theta} \mathrm{P}_{1} \omega_{\mathrm{p}}+\omega_{\mathrm{o}}^{2} \mathrm{P}_{2}\right) \cos \omega_{\mathrm{p}} \mathrm{t}+\omega_{\mathrm{o}}^{2} \mathrm{P}_{\mathrm{o}}= \\
=\frac{\mathrm{L}_{\mathrm{c}}}{\mathrm{I}_{\mathrm{z}}}+\frac{\mathrm{L}_{\mathrm{po}}}{\mathrm{I}_{\mathrm{z}}} \sin \omega_{\mathrm{p}} \mathrm{t} .
\end{gathered}
$$

Методом неопределенных коэффициентов [4] находим значения $\mathrm{P}_{\mathrm{o}}, \mathrm{P}_{1}$ и $\mathrm{P}_{2}$ :

$$
\begin{gathered}
P_{o}=\frac{L_{c}}{I_{z} \omega_{o}^{2}} ; \\
P_{1}=\frac{L_{p o}\left(\omega_{o}^{2}-\omega_{p}^{2}\right)}{I_{z}\left[\left(\omega_{o}^{2}-\omega_{p}^{2}\right)^{2}+4 v_{\theta}^{2} \omega_{p}^{2}\right]} ; \\
P_{2}=-\frac{L_{p o} 2 v_{\theta} \omega_{p}}{I_{z}\left[\left(\omega_{o}^{2}-\omega_{p}^{2}\right)^{2}+4 v_{\theta}^{2} \omega_{p}^{2}\right]} .
\end{gathered}
$$

Подставляем полученные значения коэффициентов $\mathrm{P}_{\mathrm{o}}, \mathrm{P}_{1}$ и $\mathrm{P}_{2}$ в выражение (5) и получим искомое выражение для угла рыскания:

$$
\psi_{\mathrm{r}}=\frac{\mathrm{L}_{\mathrm{c}}}{\mathrm{I}_{\mathrm{z}} \omega_{\mathrm{o}}^{2}}+\frac{\mathrm{L}_{\mathrm{po}}\left(\omega_{\mathrm{o}}^{2}-\omega_{\mathrm{p}}^{2}\right)}{\mathrm{I}_{\mathrm{z}}\left[\left(\omega_{\mathrm{o}}^{2}-\omega_{\mathrm{p}}^{2}\right)^{2}+4 v_{\theta}^{2} \omega_{\mathrm{p}}^{2}\right]} \sin \omega_{\mathrm{p}} \mathrm{t}-\frac{\mathrm{L}_{\mathrm{po}} 2 v_{\theta} \omega_{\mathrm{p}}}{\mathrm{I}_{\mathrm{z}}\left[\left(\omega_{\mathrm{o}}^{2}-\omega_{\mathrm{p}}^{2}\right)^{2}+4 v_{\theta}^{2} \omega_{\mathrm{p}}^{2}\right]} \cos \omega_{\mathrm{p}} \mathrm{t} .
$$

Преобразуем это выражение, используя амплитудно-фазовое преобразование:

где $\alpha=\operatorname{arctg} \frac{2 v_{\theta} \omega_{\mathrm{p}}}{\omega_{\mathrm{o}}^{2}-\omega_{\mathrm{p}}^{2}}$.

$$
\psi_{\mathrm{r}}=\frac{\mathrm{L}_{\mathrm{c}}}{\mathrm{I}_{\mathrm{z}} \omega_{\mathrm{o}}^{2}}+\frac{\mathrm{L}_{\mathrm{po}}}{\mathrm{I}_{\mathrm{z}}\left[\left(\omega_{\mathrm{o}}^{2}-\omega_{\mathrm{p}}^{2}\right)^{2}+4 v_{\theta}^{2} \omega_{\mathrm{p}}^{2}\right]^{1 / 2}} \sin \left(\omega_{\mathrm{p}} \mathrm{t}-\alpha\right),
$$


Обозначим $\psi_{\text {ro }}=\frac{\mathrm{L}_{\text {po }}}{\mathrm{I}_{\mathrm{z}}\left[\left(\omega_{\mathrm{o}}^{2}-\omega_{\mathrm{p}}^{2}\right)^{2}+4 v_{\theta}^{2} \omega_{\mathrm{p}}^{2}\right]^{1 / 2}}-$ амплитудное значение угла рыскания от воздействия гармонической составляющей возмущающего момента и окончательно получим:

$$
\psi_{\mathrm{r}}=\psi_{\mathrm{rc}}+\psi_{\text {ro }} \sin \left(\omega_{\mathrm{p}} \mathrm{t}-\alpha\right)
$$

где $\psi_{\mathrm{rc}}=\frac{\mathrm{L}_{\mathrm{c}}}{\mathrm{I}_{\mathrm{z}} \omega_{\mathrm{o}}^{2}}$ - постоянный угол рыскания, вызывающийся постоянной составляющей возмущающего момента.

С учетом решения соответствующего однородного уравнения:

$$
\psi_{\mathrm{ob}}=\exp \left(-v_{\theta} \mathrm{t}\right)\left[\mathrm{A}_{1} \sin \omega_{\mathrm{d}} \mathrm{t}+\mathrm{A}_{2} \cos \omega_{\mathrm{d}} \mathrm{t}\right]
$$

общее решение дифференциального уравнения (3) принимает вид:

$$
\begin{gathered}
\psi=\exp \left(-v_{\theta} t\right)\left[\mathrm{A}_{1} \sin \omega_{d} \mathrm{t}+\mathrm{A}_{2} \cos \omega_{\mathrm{d}} \mathrm{t}\right]+\psi_{\mathrm{r}}= \\
=\exp \left(-v_{\theta} \mathrm{t}\right)\left[\mathrm{A}_{1} \sin \omega_{\mathrm{d}} \mathrm{t}+\mathrm{A}_{2} \cos \omega_{\mathrm{d}} \mathrm{t}\right]+\psi_{\mathrm{rc}}+\psi_{\text {ro }} \sin \left(\omega_{\mathrm{p}} \mathrm{t}-\alpha\right),
\end{gathered}
$$

где $\mathrm{A}_{1}, \mathrm{~A}_{2}$ - постоянные интегрирования, зависящие от начальных условий.

Анализ общего решения (6) показывает, что при пропорциональном законе управления собственные затухающие колебания судна по углу рыскания дополнены гармоническими вынужденными колебаниями с частотой качки, причем имеется постоянный угол рыскания, который возникает от несимметричных рысканий в случае наличия постоянного возмущающего момента. При движении судна его рыскание относительно программной траектории движения вызывает боковое смещение судна $d$, которое определяется ортогональной относительно программной траектории составляющей скорости судна, возникающей из-за его рысканий, т. е. Vsin $\psi$ Очевидно, что величина текущего значения бокового смещения d определяется выражением:

$$
\mathrm{d}=\int_{0}^{\mathrm{t}} \mathrm{V} \sin \psi \mathrm{d} \tau
$$

Учитывая, что амплитудные значения углов рыскания судов в реальных условиях эксплуатации, как правило, не превосходят 5-7, то $\sin \psi$ можно заменить самим углом $\psi$ в радианной мере. Поэтому:

$$
\mathrm{d}=\mathrm{V} \int_{0}^{\mathrm{t}} \psi \mathrm{d} \tau
$$

где угол рыскания భ определяется выражением (6).

В свою очередь, выражение (6) можно записать в более удобном виде:

$$
\psi=\exp \left(-v_{\theta} \mathrm{t}\right) \mathrm{A} \sin \left(\omega_{\mathrm{d}} \mathrm{t}-\beta\right)+\psi_{\mathrm{rc}}+\psi_{\text {ro }} \sin \left(\omega_{\mathrm{p}} \mathrm{t}-\alpha\right)
$$

где $\mathrm{A}=\sqrt{\mathrm{A}_{1}^{2}+\mathrm{A}_{2}^{2}}, \beta=\operatorname{arctg} \frac{\mathrm{A}_{2}}{\mathrm{~A}_{1}}$.

Следовательно, выражение для бокового смещения судна d: 


$$
\mathrm{d}=\mathrm{V}\left[\mathrm{A} \int_{0}^{\mathrm{t}} \exp \left(-v_{\theta} \tau\right) \sin \left(\omega_{\mathrm{d}} \tau-\beta\right) \mathrm{d} \tau+\psi_{\mathrm{rc}} \int_{0}^{\mathrm{t}} \mathrm{d} \tau+\psi_{\mathrm{ro}} \int_{0}^{\mathrm{t}} \sin \left(\omega_{\mathrm{p}} \tau-\alpha\right) \mathrm{d} \tau\right] .
$$

После интегрирования выражения (7) получим:

$$
\begin{aligned}
& \mathrm{d}=\mathrm{V}\left\{\mathrm{A}\left(\frac{\omega_{\mathrm{d}}}{\omega_{\mathrm{d}}^{2}+v_{\theta}^{2}}\right)\{\right.\left.\cos \beta-\frac{v_{\theta}}{\omega_{\mathrm{d}}} \sin \beta-\exp \left(-v_{\theta} \mathrm{t}\right)\left[\cos \left(\omega_{\mathrm{d}} \mathrm{t}-\beta\right)+\frac{v_{\theta}}{\omega_{\mathrm{d}}} \sin \left(\omega_{\mathrm{d}} \mathrm{t}-\beta\right)\right]\right\}+ \\
&\left.+\psi_{\mathrm{rc}} \mathrm{t}+\psi_{\text {ro }} \frac{1}{\omega_{\mathrm{p}}}\left[\cos \alpha-\cos \left(\omega_{\mathrm{p}} \mathrm{t}-\alpha\right)\right]\right\} .
\end{aligned}
$$

Обращаем внимание, что величина сноса $d$ является суммой трех составляющих: собственные затухающие колебания судна по углу рыскания, растущий снос судна под действием постоянной слагаемой возмущающего момента и вынужденное колебание судна по углу рыскания, вызванное гармонической слагаемой возмущающего момента. Отметим, что величина $\mathrm{d}$ определяется влиянием возмущающего момента, т. е. второй и третей составляющими, в то время, как первой составляющей в первом приближении можно пренебречь. Поэтому для пропорционального закона управления снос $\mathrm{d}_{1}$ выражается следующим образом:

$$
\begin{gathered}
\mathrm{d}_{1}=\mathrm{V}\left\{\psi_{\mathrm{rc}} \mathrm{t}+\psi_{\text {ro }} \frac{1}{\omega_{\mathrm{p}}}\left[\cos \alpha-\cos \left(\omega_{\mathrm{p}} \mathrm{t}-\alpha\right)\right]\right\}, \text { или } \\
\mathrm{d}_{1}=\mathrm{V}\left\{\frac{\mathrm{L}_{\mathrm{c}}}{\mathrm{I}_{\mathrm{z}} \omega_{\mathrm{o}}^{2}} \mathrm{t}+\frac{\mathrm{L}_{\mathrm{po}}}{\mathrm{I}_{\mathrm{z}} \omega_{\mathrm{p}}\left[\left(\omega_{\mathrm{o}}^{2}-\omega_{\mathrm{p}}^{2}\right)^{2}+4 v_{\theta}^{2} \omega_{\mathrm{p}}^{2}\right]^{1 / 2}}\left[\cos \alpha-\cos \left(\omega_{\mathrm{p}} \mathrm{t}-\alpha\right)\right]\right\} .
\end{gathered}
$$

Для компенсации постоянного смещения от несимметричных рысканий в закон управления включается дополнительный сигнал, пропорциональный интегралу, а также используется сигнал, зависящий от угловой скорости рысканий.

В случае пропорционально-дифференциального закона управления угол кладки руля $\beta=\frac{\mathrm{k}_{1}}{\mathrm{k}_{4}} \psi+\frac{\mathrm{k}_{2}}{\mathrm{k}_{4}} \dot{\psi}$ и уравнение (2) принимает вид:

$$
\ddot{\psi}+2 v_{\theta 1} \dot{\psi}+\omega_{\mathrm{o}}^{2} \psi=\frac{\mathrm{L}_{\mathrm{c}}}{\mathrm{I}_{\mathrm{z}}}+\frac{\mathrm{L}_{\mathrm{po}}}{\mathrm{I}_{\mathrm{z}}} \sin \omega_{\mathrm{p}} \mathrm{t},
$$

где $v_{\theta 1}=\frac{1}{2}\left(\frac{\mathrm{C}_{1}}{\mathrm{I}_{\mathrm{z}}}+\frac{\mathrm{C}_{2}}{\mathrm{I}_{\mathrm{z}}} \frac{\mathrm{k}_{2}}{\mathrm{k}_{4}}\right)-$ коэффициент затухания.

В случае реализации ПИД закона управления снос $\mathrm{d}_{2}$ имеет только гармоническую составляющую с частотой рыскания:

$$
\mathrm{d}_{2}=\psi_{\mathrm{ro} 1} \frac{\mathrm{V}}{\omega_{\mathrm{p}}}\left[\cos \alpha-\cos \left(\omega_{\mathrm{p}} \mathrm{t}-\alpha\right)\right]
$$

где $\psi_{\text {ro1 }}=\frac{\mathrm{L}_{\mathrm{po}}}{\mathrm{I}_{\mathrm{z}} \omega_{\mathrm{p}}\left[\left(\omega_{\mathrm{o}}^{2}-\omega_{\mathrm{p}}^{2}\right)^{2}+4 v_{\theta 1}^{2} \omega_{\mathrm{p}}^{2}\right]^{1 / 2}}$. 
Максимальное значение $\mathrm{d}_{2 \max }$ сноса $\mathrm{d}_{2}$, вызванного гармонической составляющей возмущающего момента имеет вид:

$$
\mathrm{d}_{2 \max }=\frac{\mathrm{L}_{\mathrm{po}}}{\mathrm{I}_{\mathrm{z}} \omega_{\mathrm{p}}\left[\left(\omega_{\mathrm{o}}^{2}-\omega_{\mathrm{p}}^{2}\right)^{2}+4 v_{\theta 1}^{2} \omega_{\mathrm{p}}^{2}\right]^{1 / 2}} \frac{\mathrm{V}}{\omega_{\mathrm{p}}}\left\{1+\frac{\left(\omega_{\mathrm{o}}^{2}-\omega_{\mathrm{p}}^{2}\right)}{\left[\left(\omega_{\mathrm{o}}^{2}-\omega_{\mathrm{p}}^{2}\right)^{2}+4 v_{\theta 1}^{2} \omega_{\mathrm{p}}^{2}\right]^{1 / 2}}\right\} .
$$

В свою очередь, максимальное значение $\mathrm{d}_{1 \max }$ сноса $\mathrm{d}_{1}$ (8), вызванного гармонической составляющей возмущающего момента при пропорциональном законе управления:

$$
\mathrm{d}_{1 \max }=\frac{\mathrm{L}_{\mathrm{po}}}{\mathrm{I}_{\mathrm{z}} \omega_{\mathrm{p}}\left[\left(\omega_{\mathrm{o}}^{2}-\omega_{\mathrm{p}}^{2}\right)^{2}+4 v_{\theta}^{2} \omega_{\mathrm{p}}^{2}\right]^{1 / 2}} \frac{\mathrm{V}}{\omega_{\mathrm{p}}}\left\{1+\frac{\left(\omega_{\mathrm{o}}^{2}-\omega_{\mathrm{p}}^{2}\right)}{\left[\left(\omega_{\mathrm{o}}^{2}-\omega_{\mathrm{p}}^{2}\right)^{2}+4 v_{\theta}^{2} \omega_{\mathrm{p}}^{2}\right]^{1 / 2}}\right\} .
$$

Выражения для максимальных сносов $\mathrm{d}_{1 \max } \quad$ и $\mathrm{d}_{2 \max }$ отличаются значениями коэффициентов затухания $v_{\theta 1}$ и $v_{\theta}$, причем:

$$
v_{\theta 1}=\frac{1}{2}\left(\frac{\mathrm{C}_{1}}{\mathrm{I}_{\mathrm{z}}}+\frac{\mathrm{C}_{2}}{\mathrm{I}_{\mathrm{z}}} \frac{\mathrm{k}_{2}}{\mathrm{k}_{4}}\right) \text { и } v_{\theta}=\frac{\mathrm{C}_{1}}{2 \mathrm{I}_{\mathrm{z}}} \text {. }
$$

Очевидно, что $v_{\theta 1}>v_{\theta}$ и, следовательно, $\mathrm{d}_{1 \max }>\mathrm{d}_{2 \max }$.

\section{Выводы и перспектива дальнейшей работы по данному направлению}

Таким образом, наиболее приемлемым является ПИД закон управления, так как предупреждает появление постоянного смещения и обеспечивает минимальное значение максимальной амплитуды $\mathrm{d}_{\max }$ рысканий вынужденных колебаний. В дальнейшем целесообразно исследовать влияние стохастических факторов на точность проводки судна программой траекторией.

\section{ЛИТЕРАТУРА}

1. K. Benedict. Simulation Augmented Manoeuvring Design and Monitoring - a New Method for Advanced Ship Handling/ K. Benedict, M. Kirchhoff, M. Gluch, S. Fischer, M. Schaub, M. Baldauf, S. Klaes// TransNav, International magazine on marine navigation and safety of marine transport, Vol. 8, № 1, page 131-141, 2014.

2. C.J. Shi. Identification of Ship Maneuvering Model Using Extended Kalman Filters/ C.J. Shi, D. Zhao, J. Peng, C. Shen// TransNav, International magazine on marine navigation and safety of marine transport, Vol. 3, № 1, page 105-110, 2009.

3. M. Ljacki. Intelligent Prediction of Ship Maneuvering / M. Ljacki // International magazine on marine navigation and safety of marine transport, Vol. 10, № 3, page 511-516, 2016.

4. Пискунов Н. С. Дифференциальное и интегральное исчисление / Пискунов Н. С. - М.: Наука, 1985. - 560 с. 